\title{
Predimensionamiento gráfico multicriterio en el plano de secciones metálicas bisimétricas
}

\section{Graphical multi-criteria in-plane preliminary sizing of bisymmetrical all-metal cross-sections}

$\underline{\text { Juan José Jorquera-Lucerga }^{(*)} \text {, Juan Manuel García-Guerrero (*) }}$

\section{RESUMEN}

Se presenta y detalla un método original de predimensionamiento en el plano de secciones metálicas simétricas basado en primer lugar en un tratamiento simplificado de la geometría de la sección. Esto posibilita que cualquier criterio de predimensionamiento que necesite el proyectista, basado en la normativa y su propia experiencia, pueda ser representado gráficamente. Esta doble aproximación lleva a un predimensionamiento muy intuitivo, que identifica claramente los criterios que condicionan las dimensiones de la sección y minimiza eficazmente el peso propio de la sección transversal. El método resulta especialmente eficaz cuando se han de verificar simultáneamente varios criterios, como los relacionados con la rigidez de la sección, limitación de tensiones o rotura. Se acompaña de dos ejemplos ilustrativos y recomendaciones sobre el diseño. Además, se destaca la importancia de un adecuado criterio estructural en las primeras etapas del proyecto.

Palabras clave: Estructuras de acero; secciones transversales; predimensionamiento; diseño conceptual.

\section{ABSTRACT}

An original method of in-plane preliminary design of symmetrical steel cross-sections is shown and detailed. It is based firstly on a simplified treatment of the section geometry. This makes it possible for any pre-dimensioning criteria defined by the designer, based on standards and his/her own experience, to be represented graphically. This double approach leads to a very intuitive preliminary dimensioning, which clearly identifies the criteria that govern the sizing of the section and helps to minimize, in a very effective way, the self-weight of the cross-section. The method is particularly effective when several criteria must be verified simultaneously, such as those related to section stiffness, stress limitations or failure. It is accompanied by two illustrative examples and design recommendations. Additionally, this paper highlights the relevance of good engineering judgement in early design steps.

Keywords: Steel structures; cross-sections; preliminary design; conceptual design.

(*) Dr. Ing. Caminos, Canales y Puertos. Dpto. Ing. Minera y Civil. Universidad Politécnica de Cartagena (España) Persona de contacto/Corresponding author: juanjo.jorquera@upct.es (J.J. Jorquera-Lucerga) ORCID: http://orcid.org/oooo-0oo2-3601-4725 (Jorquera-Lucerga); http://orcid.org/oooo-ooo1-7784-0284 (García-Guerrero)

Cómo citar este artículo/Citation: Juan José Jorquera-Lucerga, Juan Manuel García-Guerrero (2021). Predimensionamiento gráfico multicriterio en el plano de secciones metálicas bisimétricas. Informes de la Construcción, 73(564): e418. https://doi.org/10.3989/ic.82283

Copyright: (C) 2021 CSIC. Este es un artículo de acceso abierto distribuido bajo los términos de la licencia de uso y distribución Creative Commons Reconocimiento 4.0 Internacional (CC BY 4.0). 


\section{INTRODUCCIÓN}

\subsection{Consideraciones sobre el predimensionamiento}

El objeto de este artículo es presentar detalladamente un método original, desarrollado por los autores, de predimensionamiento en el plano de secciones metálicas, con la intención de que resulte útil como herramienta a los proyectistas en las fases iniciales de su trabajo.

En la etapa del diseño preliminar de los elementos resistentes principales de una estructura, como la sección transversal de un puente, las vigas de un edificio o los pórticos de una nave industrial, suele suponerse que su respuesta estructural puede descomponerse en el plano y fuera de éste. Es decir, que los comportamientos resistentes en el plano de la estructura y en el perpendicular están desacoplados, y que, por lo tanto, pueden ser analizados por separado.

En este proceso, el diseño preliminar dentro del plano es generalmente el paso que más tiempo requiere, y normalmente es llevado a cabo por proyectistas con una cierta experiencia, porque un dimensionamiento preliminar preciso, aun sin perder simplicidad, puede acelerar significativamente todo el proceso de diseño.

El dimensionamiento preliminar suele basarse en el estudio de estructuras de la misma o similares tipologías, en cuyo caso las dimensiones principales de los elementos más relevantes, como el canto de las secciones transversales, suelen definirse mediante reglas sencillas, basadas en la literatura técnica y en la propia experiencia de cada proyectista. $\mathrm{Al}$ respecto, véase, por mencionar unos pocos ejemplos de muestra, la compilación de dimensiones típicas para obras de paso de carreteras que se puede encontrar en la guía editada por el Ministerio de Fomento español (1), las recomendaciones que aparecen en Lebet (2), Manterola (3) o Menn (4), o los estudios de Leonhardt (5) y Salonga y Gauvreau (6) para los puentes arco de hormigón. También se recurre, principalmente en estructuras de gran coste, complejidad o novedad, a estudios paramétricos de optimización $(7,8)$ y de sensibilidad, en los que se pretende identificar con claridad cuáles son los parámetros que definen el comportamiento de la estructura, e, incluso, ayudar a elegir entre diferentes tipologías posibles.

Una vez establecidas las dimensiones iniciales de los elementos estructurales, a efectos de predimensionamiento, es habitual considerar que la solución es válida cuando verifica algunos criterios simplificados en relación con los Estados Límites de Servicio (ELS) y Últimos (ELU) que suelen expresarse, en estructuras metálicas, como limitaciones de tensiones y de deformaciones para determinadas combinaciones simplificadas de cargas.

Estos criterios simplificados se definen por el proyectista, que toma las combinaciones consideradas en las normativas y recomendaciones, como, por ejemplo, las recogidas en la IAP (9), el CTE (10) o el EC-1 (11), y las simplifica, escogiendo sólo las acciones que considera condicionantes del diseño final, para verificar los criterios definidos en la normativa, como el EC-3 (12) y (13) o, generalmente, versiones simplificadas de los mismos.

Salvo excepciones para casos sencillos, este proceso es forzosamente iterativo, pues los esfuerzos utilizados en el pre- dimensionamiento (obtenidos, por ejemplo, con modelos sencillos de elementos finitos utilizando un software como SAP200o (14)) dependen de las características mecánicas de las secciones, que a su vez se redefinen en función de esos esfuerzos. La experiencia del proyectista reduce significativamente la duración de este proceso.

\subsection{Bases del método de predimensionamiento pro- puesto}

El método descrito en este artículo pretende precisamente ayudar al proyectista en este proceso de redefinición de las secciones, en el que lógicamente se busca minimizar el peso propio de la estructura (es decir, su coste), sin perder de vista otras consideraciones, como estéticas o constructivas. En este proceso, a veces, lleva cierto trabajo discernir cuál es el criterio determinante del predimensionamiento, situación que se complica cuando la sección transversal ha de verificar dos o más criterios simultáneamente. En ese sentido, un método como el propuesto que ayuda a distinguir los criterios relevantes de los irrelevantes resulta de mucha utilidad.

Como se justifica en la sección 2, el método descrito es general, si bien la formulación presentada y detallada se refiere a secciones bisimétricas en doble $\mathrm{T}$ y en cajón.

El método está basado en dos hipótesis principales: La primera base del método es un tratamiento simplificado de la geometría de la sección, dado que sólo interesa el comportamiento en su plano. La sección se define en función de tres variables: $\Omega$, o área total de la sección transversal; $z$, o distancia entre los centros de gravedad de las alas y $\beta$, que corresponde a la esbeltez de alma, o almas, de la sección. Esta definición simplificada se ha tomado de una publicación de Argüelles (15), si bien el cuerpo del artículo explota enormemente sus posibilidades, basado en la investigación desarrollada en (16).

La segunda base del método estriba en que este tratamiento simplificado posibilita que cualquier criterio de predimensionamiento que necesite el proyectista, basado en la normativa y su propia experiencia, pueda ser representado gráficamente en los ejes adecuados. Por lo tanto, pueden definirse, gráficamente, zonas de validez y de invalidez del mismo. El punto óptimo para varios criterios simultáneos puede fácilmente obtenerse mediante la intersección de las diferentes zonas de validez y seleccionando de toda esa zona el punto que maximice (o minimice) el valor buscado. Por supuesto, el proyectista puede elegir ese punto o cualquier otro del dominio de validez, por ejemplo, por razones estéticas o constructivas. En este artículo se ha considerado que lo que se busca es minimizar el peso de la sección transversal, dado que, en general, el coste del acero en una estructura metálica depende, directamente de su medición y su coste por kg colocado en obra. El método permite identificar claramente los criterios que condicionan las dimensiones de la sección y minimizar eficazmente el peso propio de la sección transversal.

Como se verá, el método no utiliza ningún algoritmo de optimización, como pudieran ser los genéticos $(17,18)$ o metaheurísticos (19). En su lugar, el método utiliza un enfoque muy clásico desde el punto de vista estructural, que permite una interacción muy intuitiva con el proyectista durante el proceso, que se vuelve extremadamente adaptable. Asimismo, el proyectista puede incorporar fácilmente cualquier otro 
criterio de diseño, tipológico o constructivo que necesite, de manera similar a como se implementan los criterios expuestos en el artículo.

El método resulta especialmente eficaz cuando se han de verificar simultáneamente varios criterios, como los relacionados con la rigidez de la sección, limitación de tensiones o rotura. Se acompaña de dos ejemplos ilustrativos y recomendaciones sobre el diseño. Además, se destaca la importancia de un adecuado criterio estructural en las primeras etapas del proyecto.

Por último, se llama la atención sobre el hecho de que este método, como corresponde a cualquier estrategia de predimensionamiento, no cubre todos los posibles modos de fallo de la estructura, en particular aquellos que dependen del comportamiento en el plano perpendicular de la sección, como por ejemplo el pandeo lateral, de los que el método propuesto se desentiende. Es obligación ineludible del proyectista conocer las limitaciones de los métodos que utiliza.

\subsection{Estructura del artículo}

El artículo comienza, en la sección 2, por establecer la simplificación de la geometría y de las características mecánicas de la sección transversal. En la sección 3, y en función de la formulación obtenida, se presentan varios criterios simplificados de predimensionamiento de la sección y su representación gráfica. En la sección 4 se detalla la metodología de predimensionamiento y se muestra algún ejemplo más de criterio de predimensionamiento. En la sección 5 se muestran dos ejemplos totalmente desarrollados. El artículo finaliza con una sección dedicada a conclusiones, que incluyen unas breves recomendaciones de proyecto.

\section{CARACTERÍSTICAS MECÁNICAS DE LA SEC- CIÓN SIMPLIFICADA}

Aunque se puede adaptar con mucha facilidad a otro tipo de secciones, el método se ha planteado para optimizar el área (y con ella, el peso) de secciones bisimétricas en doble $\mathrm{T}$ y en cajón rectangular. Es cierto que se pierde algo de generalidad por el hecho de que las alas sean iguales. Sin embargo, se ha obrado así porque, por un lado, el método propuesto queda perfectamente ilustrado y la formulación resultante es bastante menos farragosa, y por otro, porque las secciones bisimétricas son muy utilizadas en construcción metálica, dado que, a igualdad de área total, las alas iguales proporcionan la máxima inercia y máximos módulos resistentes. Una ventaja adicional estriba en que, si las acciones se determinan a partir de un modelo de elementos finitos con secciones bisimétricas, no se necesita cambiar la geometría del mismo entre iteraciones, puesto que los centros de gravedad de las mismas, $\mathrm{y}$, por tanto, las directrices, se conservan.

Como se mencionaba en la introducción, la primera base del método es un tratamiento simplificado de la geometría de la sección, dado que sólo interesa el comportamiento en su plano. La sección se define en función de tres variables muy intuitivas: la variable $\Omega$, o área total de la sección transversal, que permite obtener el coste sin más que multiplicar por su peso y su coste unitario; la variable $z$, o distancia entre los centros de gravedad de las alas, que resulta muy cercana al canto total de la sección, y la variable $\beta$, que corresponde a la esbeltez de alma, o almas, de la sección, que es muy relevante en la clasificación y comportamiento de las secciones metálicas. La si- guiente expresión permite la formulación para secciones cajón o doble $\mathrm{T}$ independientemente del número de almas.

$$
\text { [1] } \quad \beta \equiv \frac{\sum_{i} t_{w, i}}{h_{w}}
$$

Por otro lado, y como se muestra en la Figura 1, se supone que el área de cada ala, $\Omega_{f}$, está concentrada en el extremo del alma, es decir, se supone que el canto del alma, $z$ (o $h_{w}$ en la notación habitual en metálicas) coincide con la separación entre los centros de las alas, $h_{f}$. Como consecuencia, las cuatro secciones mostradas en la Figura 1 son equivalentes a efectos del predimensionamiento en el plano.

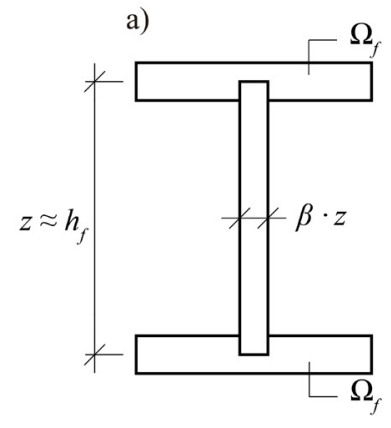

c)

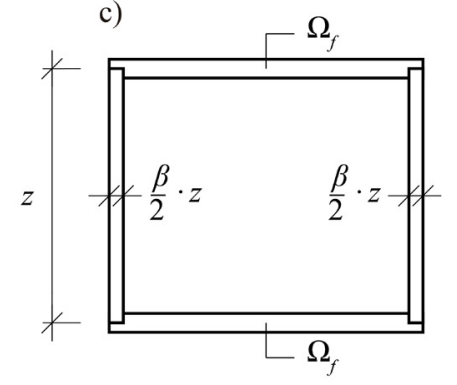

b)
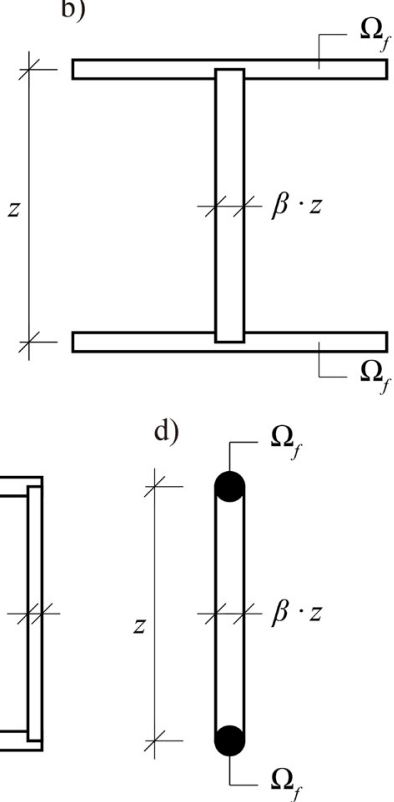

Figura 1. Secciones equivalentes $\left(z \approx h_{f}\right)$ para el predimensionamiento.

Como

$$
\text { [2] } \Omega_{f}=\frac{\Omega-\beta \cdot z^{2}}{2}
$$

para un valor dado de $\Omega$, tanto la inercia de la sección $I$, como su módulo elástico $W$, pueden expresarse como una función de $\beta$ y $z$ :

$$
\begin{gathered}
{[3] I \approx \frac{1}{12} \cdot \beta \cdot z^{4}+2 \cdot \Omega_{f} \cdot\left(\frac{z}{2}\right)^{2}=\frac{z^{2}}{2} \cdot\left(\frac{\beta \cdot z^{2}}{6}+\Omega_{f}\right)=\Omega \cdot \frac{z^{2}}{4}-\beta \frac{z^{4}}{6}} \\
{[4] W=\frac{2 \cdot I}{z}=z \cdot\left(\beta \cdot \frac{z^{2}}{6}+\frac{\Omega-\beta \cdot z^{2}}{2}\right)=\Omega \cdot \frac{z}{2}-\beta \frac{z^{3}}{3}}
\end{gathered}
$$

\subsection{Máximo modulo elástico}

En este artículo se usará el subíndice $W$ para identificar las características mecánicas correspondientes a una distribución del área que maximiza el modulo elástico. Así, el máximo módulo elástico de la sección, $W_{W}$, se obtendrá para un canto del alma $z_{W}$. La inercia correspondiente será $I_{W}$. Para un valor del área dada, el valor $z_{W}$ que maximiza $W$ se puede obtener derivando respecto de $z$ :

$$
\text { [5] } \quad \frac{d(W(z))}{d z}=0
$$

lo que resulta en:

$$
\text { [6] } \quad Z_{W}=\sqrt{\frac{\Omega}{2 \cdot \beta}}
$$


un valor que depende solo de dos magnitudes elegidas por el proyectista. Una vez conocido $z_{W}$, los valores de $I_{W}$ y de $W_{W}$ se deducen fácilmente:

$$
\begin{aligned}
\text { [7] } \quad I_{W}=\frac{\Omega^{2}}{12 \cdot \beta} \\
\text { [8] } \quad W_{W}=\frac{\Omega^{(3 / 2)}}{3 \sqrt{2 \cdot \beta}}
\end{aligned}
$$

\subsection{Máxima inercia}

Análogamente, el subíndice $I$ se usará para identificar a las propiedades mecánicas de la sección que corresponden a una distribución del área que maximiza la inercia. El máximo valor de la inercia, $I_{I}$, se obtiene para un canto del alma de $z_{I}$. El módulo elástico para esa sección es $W_{I}$. El valor de $z_{I}$ se obtiene:

$$
\begin{gathered}
\text { [9] } \frac{d(I(z))}{d z}=0 \\
\text { [10] } z_{I}=\sqrt{\frac{3 \cdot \Omega}{4 \cdot \beta}}
\end{gathered}
$$

Lo que permite obtener:

$$
\begin{aligned}
{[11] \quad I_{I} } & =\frac{3 \cdot \Omega^{2}}{32 \cdot \beta} \\
{[12] \quad W_{I} } & =\frac{\sqrt{3}}{8} \cdot \frac{\Omega^{3 / 2}}{\sqrt{\beta}}
\end{aligned}
$$

\subsection{Consideraciones sobre la sección simplificada}

La Figura 2 es útil para clarificar la notación introducida hasta ahora, pues muestra $W$ e $I$ en función de $z$ para diferentes valores de $\beta$. Todas las curvas se han obtenido para el mismo valor de $\Omega$, lo que evidencia lo relevante que puede llegar a ser el efecto de $\beta$ sobre los valores de las características mecánicas de la sección.

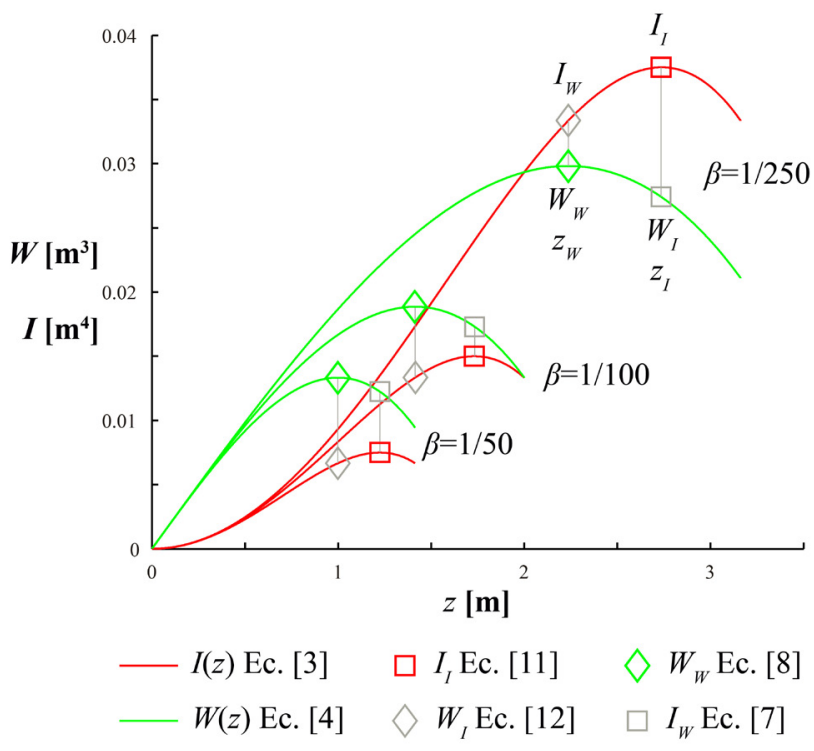

Figura 2. I(z) y $W(z)$ para diferentes $\beta$ y para un valor dado de $\Omega$.

Es de destacar que siempre se verifica que $z_{I}>Z_{W}$. De las ecuaciones [6] y [10]:

$$
\text { [13] } \quad z_{I}=\sqrt{\frac{3}{2}} \cdot z_{W} \cong 1.225 \cdot z_{W}
$$

El valor máximo posible de $z$ es $z_{\max }$, que corresponde a una distribución de área en la que toda el área de la sección está en el alma y el área de las alas es nula, lo que lleva a

$$
\text { [14] } 0<z \leq z_{\max }=\sqrt{\frac{\Omega}{\beta}}
$$

por lo que, para las secciones definidas en este artículo, los valores de $z$ mayores que $z_{\max }$ carecen de significado físico.

Análogamente, de las fórmulas anteriores, las relaciones siguientes entre las inercias y módulos cuando se maximiza $I$ o $W$ pueden resultar útiles para el predimensionamiento:

$$
\begin{aligned}
\text { [15] } \quad I_{I} & =\frac{36}{32} \cdot I_{W}=1.125 \cdot I_{W} \\
{[16] \quad W_{W} } & =\frac{8}{3 \sqrt{6}} \cdot W_{I} \approx 1.089 \cdot W_{I}
\end{aligned}
$$

Una consecuencia muy importante es que, para el mismo valor de $\Omega$, crecen $I$ y $W$ a medida que $\beta$ decrece, puesto que, si bien el área del alma se mantiene constante, el brazo mecánico de las alas crece.

La Figura 3 muestra las isolíneas de $I_{I}$ and $W_{W}$, respectivamente, para diferentes valores de $\Omega$ y $\beta$. Los valores más altos de $I$ y de $W$ siempre se obtienen, lógicamente, para las almas más esbeltas.
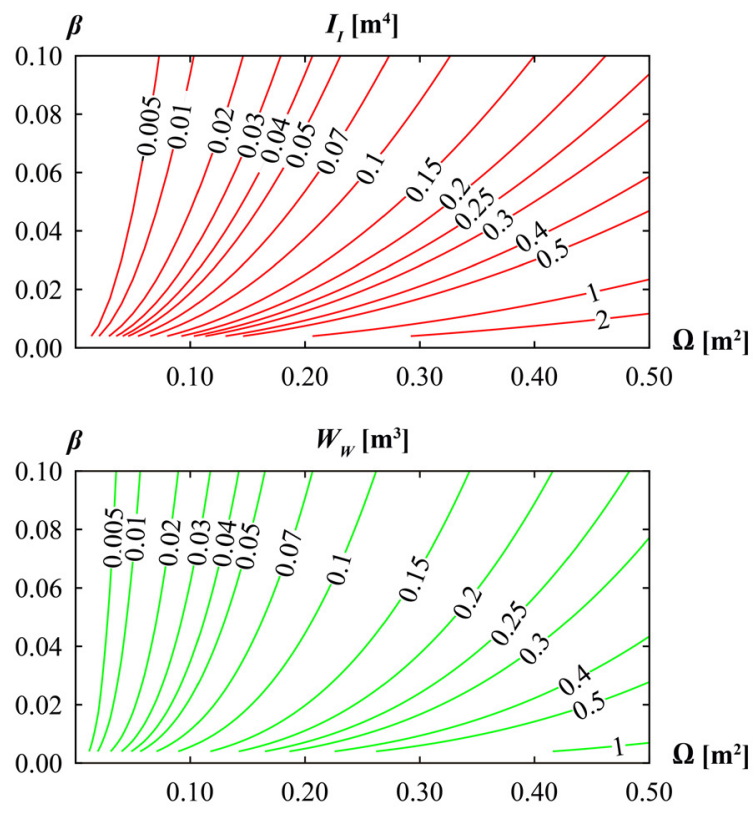

Figura 3. Isolíneas de $I_{I}[11]$ y $W_{W}[8]$

\subsection{Bondad de la simplificación}

Para mostrar la bondad de la formulación simplificada, los valores de $I_{I}$ y $W_{W}$, definidos respectivamente en las ecuaciones [11] y [8], se han dibujado y comparado con la misma formulación sin simplificaciones. Para este segundo cálculo se ha supuesto que el área de las alas, $\Omega_{f}$, se ha distribuido de dos maneras: la primera es un ala exenta con una relación $b / t_{f}=15$, mientras que la segunda es una sección cajón hueca con una relación $b / t_{f}=42$. El primer caso es aproximadamente un límite entre las clases 1 y 2 para un ala exenta en una sección armada, pues $b \approx t_{w}+2 \cdot 9 \cdot \varepsilon \cdot t_{f}$ 
$\approx 15 \cdot t_{f}$, para $f_{y}=355$ MPa y $\varepsilon=0.81$, de acuerdo con el EC-3 (12). El segundo caso corresponde a un límite para clase 3, no demasiado estricto, para un ala interna comprimida. $b \approx 42 \cdot \varepsilon \cdot t_{f}$, para $f_{y}=235$ MPa y $\varepsilon=1.00$. Los resultados se muestran en la Figura 4.

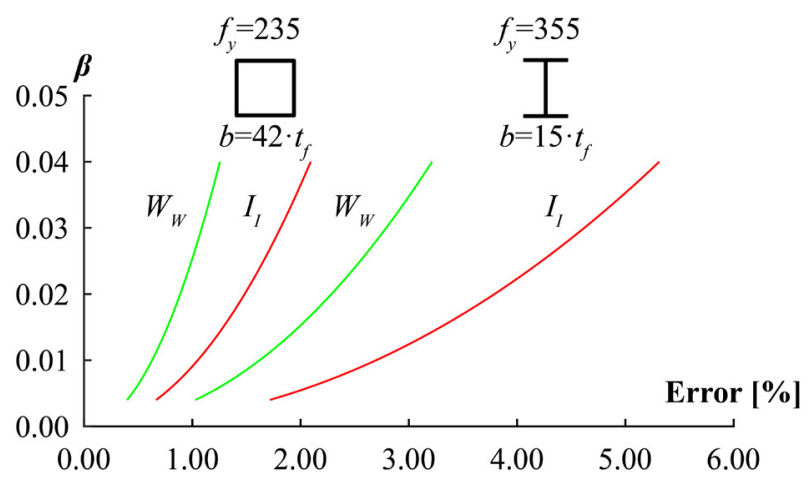

Figura 4. Error de la formulación simplificada para dos ejemplos de secciones transversales de clase 1 en doble T y de clase 3 en cajón.

Una conclusión muy práctica es que el error sólo depende de $\beta$. Para un valor dado de $\Omega_{f}$, la precisión de la formulación se incrementa según decrece $t_{f}$, lo que resulta lógico pues la distancia entre los centros de las alas se acerca más al canto total. En los casos estudiados, el error permanece por debajo del $3 \%$ para $W_{W}$, y es necesario proyectar un alma con una esbeltez de 1/30 para encontrar una sección con un error del $5 \%$ en $I_{I}$. Esta precisión se considera suficiente para un diseño preliminar, y, en cualquier caso, el error puede ser fácilmente contrarrestado utilizando en el predimensionamiento valores ligeramente más conservadores para las flechas o tensiones admisibles. El error mostrado en la Figura 4 desaparece, en la práctica, si se obtienen las características mecánicas de la sección respecto de las fibras medias de las alas.

\section{EJEMPLOS DE CRITERIOS DE PREDIMENSIO- NAMIENTO}

El proceso de dimensionamiento está basado en que cada criterio a verificar por la sección se puede dibujar en los ejes adecuados y define, gráficamente, zonas de validez y de invalidez del mismo. El punto óptimo puede obtenerse mediante la intersección de las diferentes zonas de validez para los diferentes criterios y seleccionando de toda esa zona el punto que maximice (o minimice) el valor buscado. En este artículo se ha considerado que lo que se busca es minimizar el peso de la sección transversal, dado que, en general, el coste del acero en una estructura metálica depende, directamente de su medición y su coste por kg colocado en obra. Así pues, en este artículo se ha elegido como eje de abscisas $\Omega$, o área total de la sección transversal, que permite obtener el coste sin más que multiplicar por su peso y su coste unitario.

Para el eje de ordenadas se ha elegido la variable $z$, que resulta muy cercana al canto total de la sección y que además de su carácter muy intuitivo, es muy importante desde el punto de vista visual, sin contar que muchos criterios de predimensionamiento están basados en la relación canto/luz, lo que permite contrastar con facilidad el método propuesto en este artículo con otros criterios ya conocidos por el proyectista.

La tercera variable que define la sección trasversal es la esbeltez del alma, $\beta$, que en estructuras metálicas resulta un valor crucial a la hora del dimensionamiento, pues sirve, por ejemplo, como criterio de clasificación de la sección al establecer la clase del alma, o como criterio para discernir si ha de considerarse la abolladura del alma en el cálculo de su resistencia a cortante. El valor de $\beta$ suele aparecer, además, con frecuencia en criterios de predimensionamiento de secciones metálicas. Véanse, por ejemplo, las recogidas en (1), o en las RPM-95 (20) y RPX-95 (21).

Por lo tanto, en este método, se expresarán todos los resultados en ejes $\Omega-z$ para un valor $\beta=$ cte.

\subsection{Criterio de rigidez}

El criterio de rigidez se basa en limitar la deformación, para que resulte menor que la flecha admisible $\delta_{\max }$, bajo una combinación de cargas simplificada en ELS, que llamaremos por ejemplo $q_{k}$ :

$$
\text { [17] } \quad \delta_{\max } \leq \delta(E L S)=\delta\left(q_{k}\right)
$$

Para representar este criterio gráficamente basta con dibujar la isolínea $I=I_{\min }$, donde $I_{\min }$ es el valor de la inercia que ha de tener la sección transversal para verificar el ELS de deformaciones. Por ejemplo, si se está predimensionando una viga biapoyada, y se quiere limitar el valor de la flecha máxima a $\delta_{\max }$ en el centro de la luz bajo una carga $q_{k}$ uniformemente distribuida, $I_{\min }$ se obtendría de la expresión:

$$
\text { [18] } \quad \delta_{\max } \leq \frac{5 \cdot q_{k} \cdot L^{4}}{384 \cdot E \cdot I_{\min }}
$$

Como en cualquier otro criterio de predimensionamiento, $\mathrm{y}$ dado que $q_{k}$ corresponde a una combinación simplificada de cargas, que no considerará todas las acciones posibles, $\delta_{\max }$ no ha de coincidir necesariamente con el valor máximo estricto que especifique la normativa, sino que es un valor cercano y menor que éste, definido por la experiencia del proyectista.

En la Figura 5 se representan diferentes isolíneas $I=I_{\min }$, de valores $I=0.001,0.002$ y 0.005 , en ejes $\Omega$-z para un valor $\beta$ $=1 / 100$. Es importante señalar que todas las isolíneas quedan truncadas superiormente por la curva $z=Z_{\max }$. Esto ocurre porque en esa curva toda el área de la sección está en el alma y por encima de esa curva la isolínea no tiene sentido físico. Por lo tanto, la zona de validez $I \geq I_{\min }$ será la zona del diagrama a la derecha de la curva, limitada inferior y superiormente, respectivamente por las curvas $Z=O$ y $Z=Z_{\max }$.

La expresión de la isolínea, para valores conocidos de $I_{\min }, \beta$ y $z$, viene dada por:

$$
\text { [19] } \Omega=\frac{4 \cdot I_{\min }}{z^{2}}+\frac{2}{3} \beta z^{2}
$$

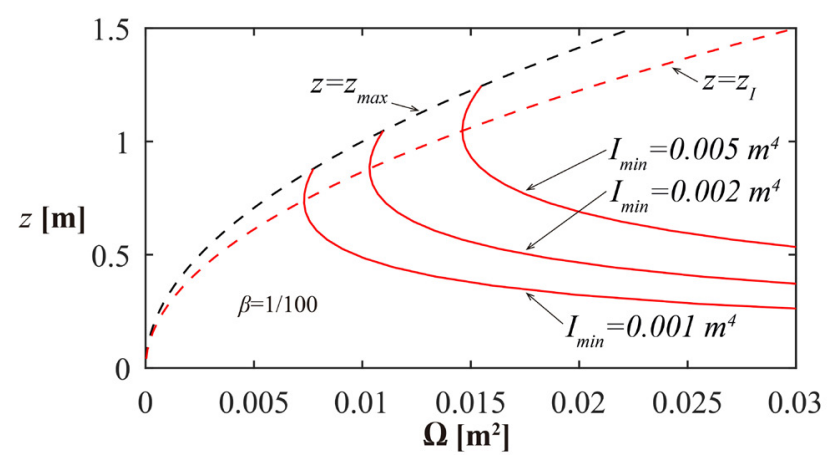

Figura 5. Isolíneas de inercia $I=0.001,0.002$ y 0.005, para $\beta=1 / 100$. 
Como puede verse, los puntos de área mínima de cada isolínea de inercia pertenecen todos a la curva $z=Z_{I}[10]$, lo que significa que, si el único criterio de selección de la sección fuera la inercia, el punto de peso (área) mínimo viene dado por la intersección de la curva $z=Z_{I}$ con la isolínea $I=I_{\min }$.

Es interesante señalar que, al contrario de lo que pudiera parecer intuitivo, para un valor dado de $\Omega$, el aumento de canto no produce un aumento de la inercia indefinidamente, sino que disminuye a partir de $z_{I}$ entre las isolíneas $z=Z_{I}$ y $Z=Z_{\max }$.

\subsection{Criterio de limitación de la tensión normal}

De un modo similar al criterio anterior, el criterio tensional consiste en limitar la tensión normal máxima bajo una combinación de cargas simplificada en ELU, que llamaremos por ejemplo $q_{d}$, para que resulte menor que la tensión admisible:

$$
\text { [20] } \sigma_{\max } \leq \sigma(E L U)=\sigma\left(q_{d}\right)
$$

Este criterio se reduce a obtener la isolínea $\sigma=\sigma_{\max }$, en la que las coordenadas $(\Omega, z)$ de cada punto se obtienen resolviendo la ecuación:

$$
\text { [21] } \quad \sigma_{\max }=\frac{N\left(q_{d}\right)}{\Omega}+\frac{M\left(q_{d}\right)}{W(\Omega, \beta, z)}
$$

donde $N$ y $M$ corresponden a los esfuerzos obtenidos para la combinación simplificada de acciones y $W$ se evalúa mediante la ecuación [4]. Por la simetría de la sección transversal, el signo de $M$ es indiferente. Esta expresión puede ser utilizada también como criterio de predimensionamiento en ELS, por lo que las acciones a considerar en cada caso, así como sus coeficientes de ponderación, deberán adaptarse según la normativa y la experiencia del proyectista.

Las consideraciones acerca del valor de $\delta_{\max }$ siguen siendo válidas al hablar de $\sigma_{\max }$, pues no ha de coincidir exactamente con el valor máximo estricto que especifique la normativa (por ejemplo, para ELU, $\sigma_{\max }=f_{\mathrm{y}} / \gamma_{\mathrm{Mo}}$ ), sino que será un valor cercano y menor que éste, nuevamente definido por la experiencia del proyectista.

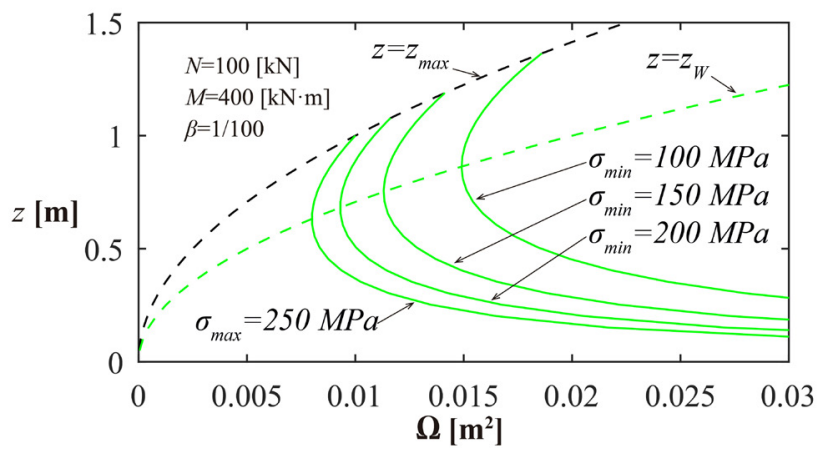

Figura 6. Isolíneas tensionales para $\sigma_{\max }=100,150,200$ y $250 \mathrm{MPa}$, $\operatorname{para} \beta=1 / 100$.

En la Figura 6 se representan diferentes isolíneas, $\sigma=\sigma_{\max }$ de valores $\sigma_{\max }=100,150,200$ y $250 \mathrm{MPa}$, en ejes $\Omega$-z para un valor $\beta=1 / 100$, y para una pareja de esfuerzos $N=100 \mathrm{kN} \mathrm{y}$ $M=400 \mathrm{kN} \cdot \mathrm{m}$. Como en el caso anterior, la zona de validez del criterio definido por la isolínea será la zona del diagrama a la derecha de la curva, limitada inferior y superiormente, respectivamente, por las curvas $z=0$ y $z=Z_{\max }$. En el caso de que $M=0$, las isolíneas degeneran en líneas verticales de valor $\Omega=N / \sigma_{\max }$.
Los puntos de área mínima de cada isolínea pertenecen todos a la curva $Z=Z_{W}[6]$, y el punto de peso mínimo está en la intersección de la curva $Z=Z_{W}$ con la isolínea $\sigma=\sigma_{\max }$.

De forma similar al caso anterior, por encima de un valor umbral, $z_{W}$, el aumento de canto provoca una disminución leve del módulo resistente entre las isolíneas $z=Z_{W}$ y $Z=Z_{\max }$.

\section{METODOLOGÍA DE PREDIMENSIONAMIENTO}

Cuando se pretende en el predimensionamiento que la sección transversal verifique simultáneamente dos o más criterios, el criterio general es que el punto solución, en un diagrama $\Omega-z$, es el punto que queda más a la izquierda (es decir, el punto de menor área) de la intersección de las zonas de validez de todos los criterios considerados.

Para ilustrar el método se supondrá que se busca minimizar el peso de la sección transversal verificando simultáneamente los dos criterios recién mostrados, el de rigidez y el de máxima tensión normal. Estos dos criterios juntos suelen ser muy usados como criterios de predimensionamiento en la práctica, según la experiencia profesional de los autores.

En la Figuras 7 a 10 se representan los cuatro casos posibles que ilustran las diferentes posibilidades. Los resultados corresponden a un código en Matlab (22) desarrollado por los autores. Para los cuatro casos posibles se ha buscado el punto de intersección de la isolínea $I=0.015 \mathrm{~m}^{4}$ con las cuatro isolíneas tensionales, $\sigma_{\max }=320,280,230$ y $200 \mathrm{MPa}$, para $N=1000 \mathrm{kN}$ y $M=4000 \mathrm{kN} \cdot \mathrm{m}$. El contorno de la zona de validez se ha marcado en color azul, y se ha marcado en éste el punto de área mínima del contorno de la zona de validez.

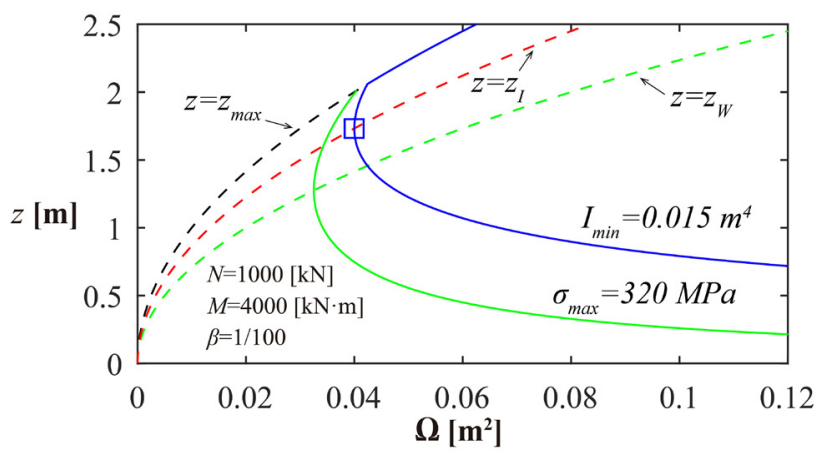

Figura 7. Intersección de isolíneas de inercia $\left(I=0.015 \mathrm{~m}^{4}\right)$ $\mathrm{y}$ tensional $\left(\sigma_{\max }=320 \mathrm{MPa}\right)$ sobre $z=Z_{\max }$

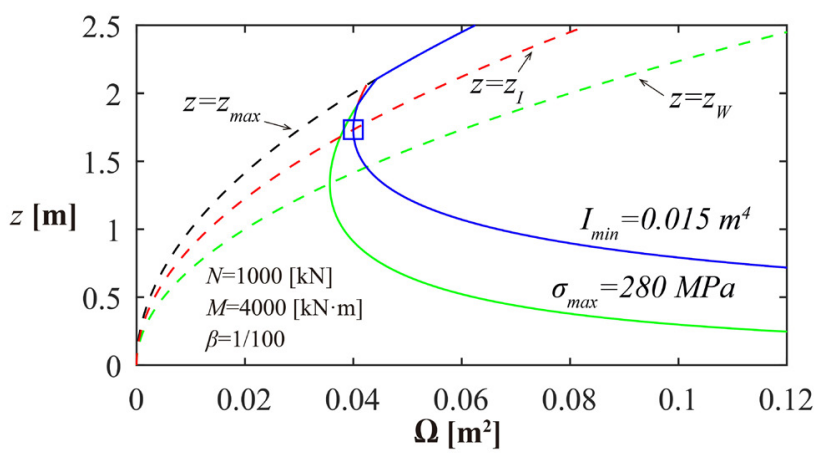

Figura 8. Intersección de isolíneas $\left(I=0.015 \mathrm{~m}^{4}\right.$ y $\left.\sigma_{\max }=280 \mathrm{MPa}\right)$ entre $Z=Z_{I}$ y $Z=Z_{\max }$ 


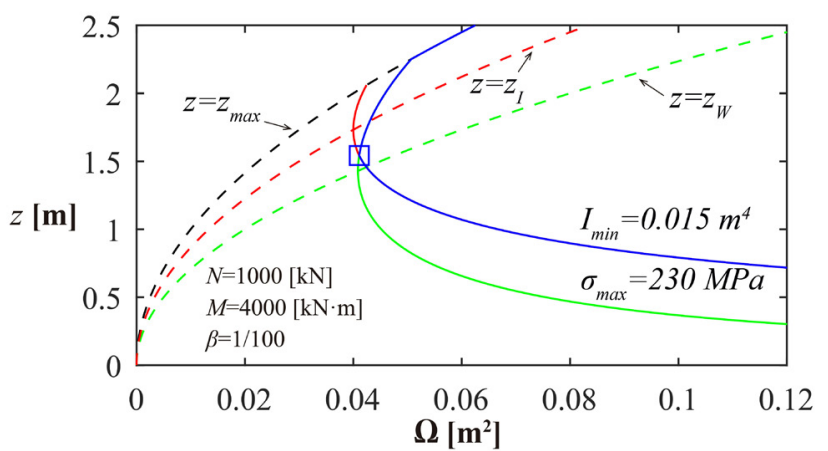

Figura 9. Intersección de isolíneas $\left(I=0.015 \mathrm{~m}^{4}\right.$ y $\left.\sigma_{\max }=230 \mathrm{MPa}\right)$ entre $Z=Z_{W}$ y $Z=Z_{I}$

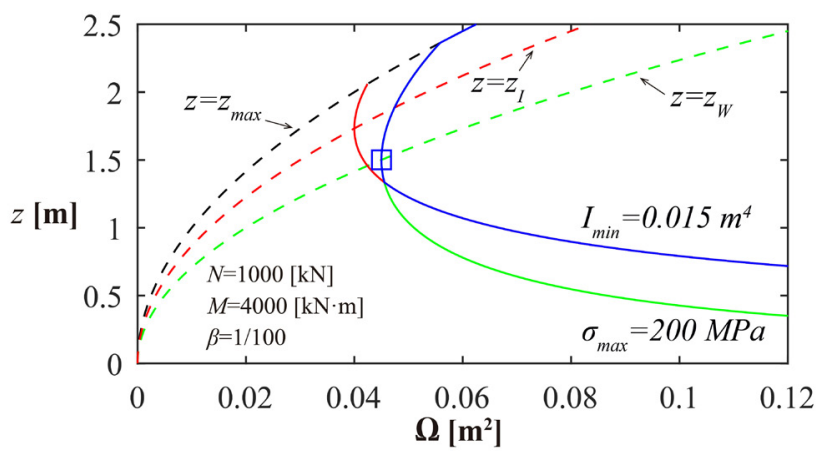

Figura 10. Intersección de isolíneas $\left(I=0.015 \mathrm{~m}^{4}\right.$ y $\left.\sigma_{\max }=200 \mathrm{MPa}\right)$ por debajo de $z=Z_{W}$

Por supuesto, todas las curvas (y sus dominios de validez asociados) quedan limitadas inferiormente por la curva $\mathrm{z}=\mathrm{O}$ y superiormente por la $Z=Z_{\max }$. En la Figura 7 , la intersección de las isolíneas tensional, $\sigma=\sigma_{\max }$, y de inercia, $I=I_{\min }$, queda por encima de esta curva, con lo que, en la práctica, es como si no se cortaran y la zona de validez de ambos criterios coincide con la zona de validez del criterio de rigidez, pues toda la isolínea de inercia queda más a la derecha. En este caso, el punto válido más a la izquierda es el punto intersección de la $Z=Z_{I}$ con la curva $I=I_{\min }$.

En la Figura 8 el punto de intersección de ambas isolíneas queda entre $z=Z_{I}$ y $z=Z_{\max }$. El dominio de validez de ambos criterios simultáneamente será una zona limitada a la izquierda por la isolínea tensional por encima del punto de intersección y por la isolínea de inercias por debajo de éste. En este caso el punto mínimo también coincide con el punto $Z=Z_{I}$ de la isolínea $I=I_{\min }$.

El caso de la Figura 9 es el más interesante puesto que es un punto que no es inmediato de deducir analíticamente. Al depender de dos criterios de predimensionamiento simultáneamente, en este caso la intersección de las isolíneas se produce entre las líneas $Z=Z_{I}$ y $Z=Z_{W}$.

Por último, en la Figura 10, la intersección de ambas isolíneas se produce bajo la curva $Z=Z_{W}$. De modo similar a los casos anteriores, el punto de peso mínimo viene dado por la intersección de la isolínea tensional con la curva $z=Z_{W}$.

Es interesante reflexionar sobre la distribución de material en la sección: mientras que en los dos primeros casos (Figuras 7 y 8) se distribuye para maximizar la rigidez, en el cuarto (Figura 10) se maximiza el módulo elástico de la sección.
El tercer caso (Figura 9) es el más interesante, puesto que el material se distribuye de una manera tal que no maximiza ni uno ni otro, sino que minimiza el peso propio de la sección mientras verifica simultáneamente ambos criterios.

Es digno de mencionarse que, dado que las isolíneas pueden degenerar en líneas (pseudo)verticales para valores de $M$ muy bajos, puede ocurrir que las isolíneas tensional y de inercias se intersecten dos veces como en la Figura 11.

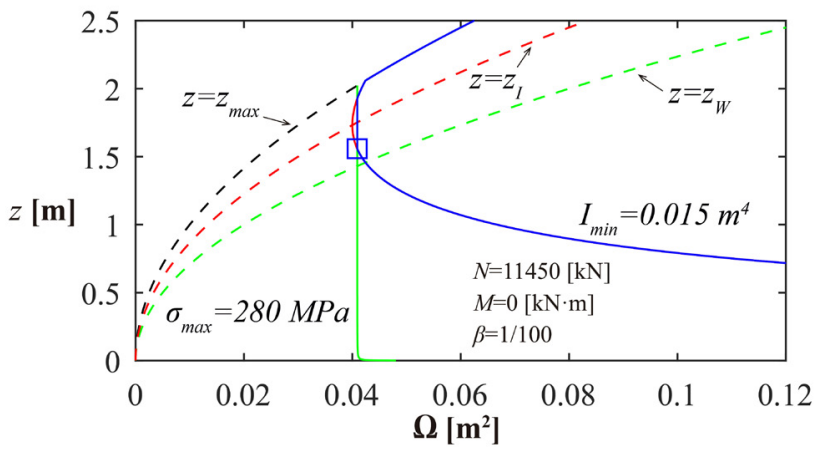

Figura 11. Intersección de isolíneas $\left(I=0.015 \mathrm{~m}^{4}\right.$ y s $\left.\mathrm{s}_{\max }=280 \mathrm{MPa}\right)$ entre $Z=Z_{I}$ y $Z=Z_{\max }$, para $N=11450 \mathrm{kN}, \mathrm{M}=0$.

En este caso, obtenido para $N=11450 \mathrm{kN}, M=0$ y $\sigma_{\max }=280$ MPa puede verse cómo el punto de mínimo peso se ha elegido correspondiendo con la mínima $z$, si bien la rama vertical a la izquierda de la zona de validez da toda ella áreas que, en la práctica, son iguales.

\subsection{Criterio de limitación de cortante máximo}

Este criterio se introduce, entre otros posibles, para ilustrar cómo en la práctica, cualquier criterio deseado por el proyectista es expresable simplificadamente en términos de $\Omega, \beta$ y z. Además, muestra cómo a los dos criterios mostrados en la sección 3 pueden añadirse todos los criterios que se deseen.

Cuando no es necesario considerar la abolladura en la resistencia al cortante del alma, ésta puede evaluarse mediante la siguiente expresión para dimensionamiento plástico extraída del EC3-1 (12), relativa a secciones de vigas armadas soldadas en I, en $\mathrm{H}$ y en cajón con carga paralela al alma:

$$
\text { [22] } \quad V_{p l . R d}=\eta \cdot \Sigma\left(h_{w} t_{w}\right) \cdot \frac{f_{y}}{\sqrt{3} \cdot \gamma_{M 0}}
$$

y que, considerando $\eta=1$ conservadoramente, coincide con la fórmula para dimensionamiento elástico del $\mathrm{EC}_{3}$-1 en secciones en I o $\mathrm{H}$, siempre que $A_{f} / A_{w} \geq 0.6$, lo que permite considerar una distribución plástica de tensiones.

Con la notación empleada en este artículo, en la que el área del alma puede expresarse como $\beta \cdot \mathrm{z}^{2}$ la fórmula anterior queda

$$
\text { [23] } \mathrm{V}=\beta \cdot z^{2} \cdot \frac{\sigma_{\max }}{\sqrt{3}}
$$

donde $V$ es el cortante considerado en el predimensionamiento, lo que resulta en el criterio, en ejes $\Omega$-z:

$$
\text { [24] } \quad z \geq \sqrt{\frac{\mathrm{V} \cdot \sqrt{3}}{\sigma_{\max }}}
$$

que es una línea horizontal por encima de la cual la sección verifica la resistencia a cortante. 
En la Figura 12 se muestra cómo afecta al dimensionamiento ya mostrado en la Figura 10 cuando además se introduce como criterio la actuación de un cortante de $V=1500 \mathrm{kN}$. En ella puede verse, en el extremo derecho, una rama horizontal, por debajo de la cual no se verifica la resistencia a cortante, y que restringe las soluciones válidas.

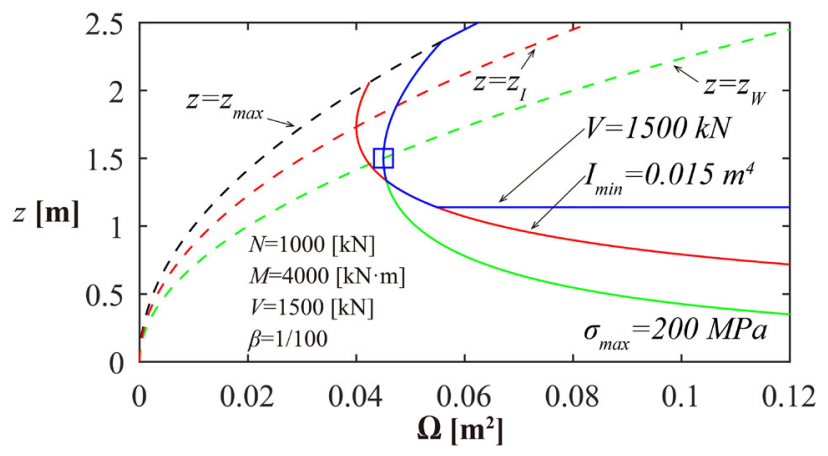

Figura 12. Diagrama de predimensionamiento de la Figura 10, al que se ha añadido el criterio de cortante máximo.

\section{EJEMPLOS DE APLICACIÓN}

\subsection{Ejemplo de aplicación $\mathrm{n}^{\mathbf{0}} \mathbf{1}$}

Para ilustrar la aplicabilidad del método se va a resolver el siguiente ejemplo: Se trata de predimensionar con el método descrito en este artículo una viga biapoyada en doble T simétrica, de $8 \mathrm{~m}$ de luz, construida en acero S 275. La limitación de flecha es $\delta_{\max } \leq L / 400$ bajo unas cargas en servicio simplificadas equivalentes a una sobrecarga uniformemente distribuida $q_{k}=12 \mathrm{kN} / \mathrm{m}$. La tensión máxima longitudinal es de $\sigma_{\max } \leq 220 \mathrm{MPa}$, bajo unas acciones mayoradas simplificadas equivalentes a una carga uniformemente repartida $q_{d}=20 \mathrm{kN} / \mathrm{m}$. La esbeltez del alma es de $1 / 60$, que está por debajo de la esbeltez $h_{w} / t_{w}$ para la que, según el EC3 y para S275 (que es de $72 \cdot \varepsilon / h=72 \cdot 092 / 1$.oo $=66.2$ ) es necesario considerar la abolladura del alma en su resistencia a cortante.

El predimensionamiento considerará los siguientes criterios: deformabilidad máxima en ELS, tensión máxima longitudinal (momento último en régimen elástico) en centro de vano y máximo cortante en ELU en apoyos.

Los esfuerzos actuantes son $M=160 \mathrm{kN} \cdot \mathrm{m}$ y $V=80 \mathrm{kN} \cdot \mathrm{m}$. Por otro lado, la inercia mínima de la sección, al aplicar la formulación para la flecha en el centro de vano de una viga simplemente apoyada (véase [18]) resulta $I_{\min } \geq 1.6 \cdot 10^{-4} \mathrm{~m}^{4}$.

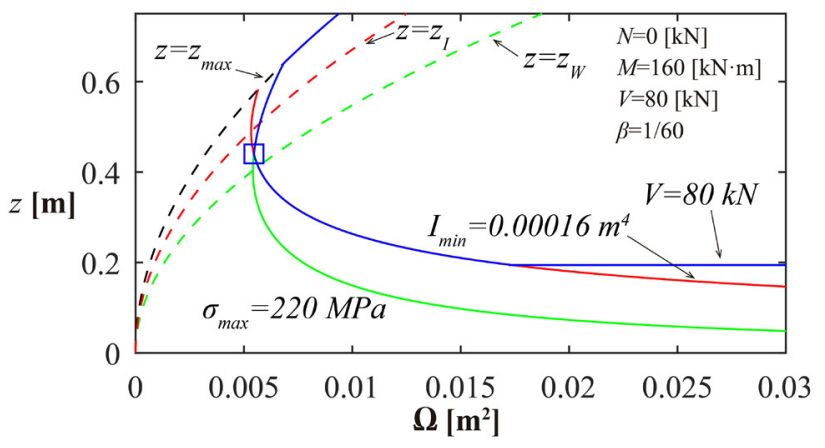

Figura 13. Ejemplo de predimensionamiento.
En la Figura 13 se muestra el diagrama del predimensionamiento con los diferentes criterios. Resulta muy interesante el resultado puesto que, en este caso, tanto el criterio de rigidez mínima como el de tensión longitudinal máxima resultan determinantes. Este caso en particular no resulta fácil de determinar analíticamente.

El resultado corresponde al punto $z=0.439 \mathrm{~m} \mathrm{y} \Omega=5 \cdot 459 \cdot 10^{-3}$ $\mathrm{m}^{2}=54.6 \mathrm{~cm}^{2}$. Tomando estos valores estrictos, los resultados son prácticamente exactos para los valores de $I_{\min }$ y de $\sigma_{\max }$.

Tomando un valor redondeado de $z=440 \mathrm{~mm}$, el área del alma resulta $\Omega_{w}=\beta \cdot Z^{2}=44^{2} / 60=32.27 \mathrm{~cm}^{2}$. El espesor del alma será de $440 / 60=7.33 \mathrm{~mm}$. Suponiendo el espesor del ala $t_{f}=10 \mathrm{~mm}, \mathrm{y}$ considerando el solape entre alma y ala de $0.5 \cdot t_{w} \cdot t_{f}$, pues $z$ es la distancia entre centros de las alas, su área resulta $\Omega_{f}=0.5 \cdot(54.6$ $32.27+0.733 \cdot 1)=11.53 \mathrm{~cm}^{2}$, dispuestas en platabandas de $115 \mathrm{x}$ $10 \mathrm{~mm}$. Como $z=440 \mathrm{~mm}$, el canto total de la viga es de $450 \mathrm{~mm}$, quedando finalmente un alma de $430 \times 8 \mathrm{~mm}$, tras redondear $t_{w}$ al espesor superior comercial más cercano.

El área total de la sección resultado queda, recién obtenido del método, de $2 \cdot 11.53 \cdot 1+43 \cdot 0.733=54.58 \mathrm{~cm}^{2}$, mientras que, si se incluyen los redondeos de alma y alas, es de $2 \cdot 11.5 \cdot 1+43 \cdot 0.8=57.4 \mathrm{~cm}^{2}$. El resultado será tanto más preciso cuanto más se acerque el espesor del alma teórico a un espesor de chapa comercial.

Para comprobar los resultados, la inercia de la sección resulta $I=1 / 12 \cdot\left(11.5 \cdot 45^{3}-10.7 \cdot 43^{3}\right)=16400 \mathrm{~cm}^{4}$, con un error de $2.7 \%$ sobre $I_{\min }=16000 \mathrm{~cm}^{4}$, debido al redondeo del espesor del alma, y que desaparece $\left(I_{\min }=15990 \mathrm{~cm}^{4}\right)$ si se considera su espesor teórico. Por otra parte, la tensión longitudinal máxima es de $\sigma=160 \cdot 1000 \cdot 22.5 / 16400=219.5 \mathrm{MPa}$, casi coincidente con el objetivo $\sigma_{\max } \leq 220 \mathrm{MPa}$. Los resultados mostrados muestran que el método muestra suficiente precisión para ser usado en el predimensionamiento.

\subsection{Ejemplo de aplicación $n^{0} 2$}

En el siguiente ejemplo, que además evidencia la versatilidad del método, se muestra cómo se puede incorporar a los criterios ya mostrados, un criterio adicional de clasificación de la sección transversal. En realidad, el criterio se refiere a la clasificación del alma, pues la clasificación de las alas mostrada en la tabla 5.2 del EC3-1.1 (12) no es posible con la notación simplificada de la Sección 2 al considerar sólo el área de las alas y no sus dimensiones. Sin embargo, la clase de las alas en voladizo (Figura 14) no condiciona por sí misma el diseño, pues nada impide en principio al proyectista distribuir sus áreas en dimensiones que verifiquen los criterios anchura/ espesor $(c / t)$ correspondientes a la clase elegida. Incluso en las alas interiores se está en el mismo caso del ala en voladizo si el ancho es opcional. Sólo en el caso de que las alas sean de ancho fijo puede su clase volverse condicionante del diseño, al venir el espesor dado como resultado del predimensionamiento. En una sección doble T como la de este ejemplo, el problema de la clase de las alas puede ser ignorado.

Sin embargo, para el alma, los criterios anchura/espesor expresados como la relación $c / t$, sí son calculables con la notación simplificada de la sección 2, para clases 1, 2 ó 3. Como durante todo el artículo se ha supuesto que la sección es, como poco, de clase 3 (dado que el criterio tensional responde a una dis- 

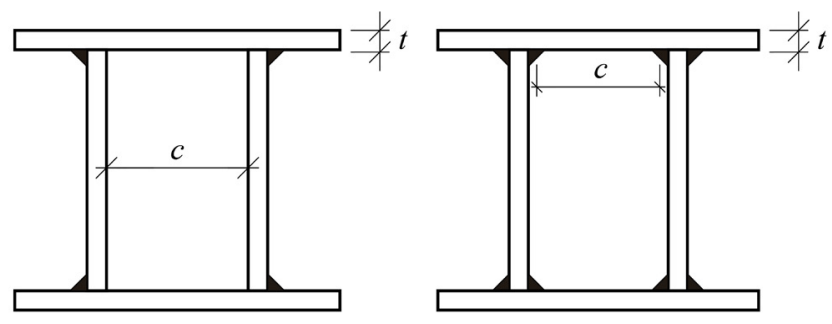

Figura 14. Alas en voladizo e interiores.

tribución elástica de tensiones y que toda el área de la sección es eficaz), en este ejemplo se obtienen, de una manera similar a las de los otros criterios de predimensionamiento ya mostrados, las isolíneas de los valores mínimos de $t / c$ para los que el alma de la sección es de clase 3 (Figura 16). Se muestra, en lugar del valor $c / t$ de la tabla 5.2 , su inverso $t / c$ porque facilita la comparación con la esbeltez del alma definida en la sección 2 (Figura 15-a), $\beta=t_{w} / z \approx t_{w} / h_{f}$. Para la formulación de las isolíneas en los casos de axil y flector simultáneos se supone que la relación $N / M$ en los esfuerzos finales de dimensionamiento, que son desconocidos en esta fase del proyecto, no cambia respecto a la de los esfuerzos de predimensionamiento, lo que es tanto como afirmar que $\psi$ (Figura 16) se mantiene constante.

a)

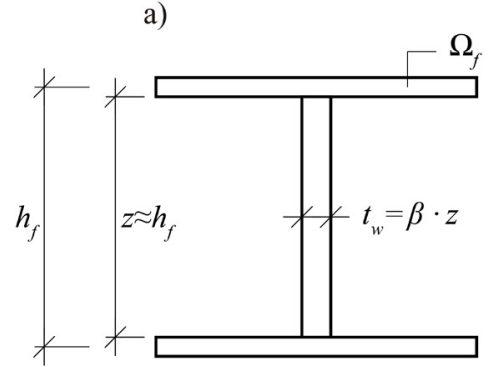

b)

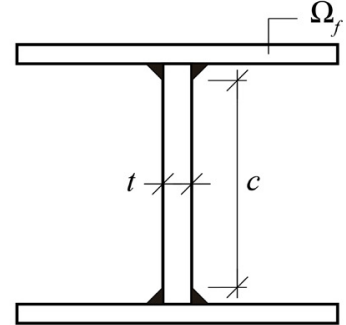

Figura 15. Definición de alma en notación simplificada y en EC3 (12).

La utilidad de las curvas $t / c=$ cte desde el punto de vista del predimensionamiento es clara, pues lo que interesa (por supuesto, si el resto de criterios lo permite) es que $\beta$, que se define a priori por el proyectista, sea igual o ligeramente superior al valor de $t / c$ mínimo para que la sección sea de clase 3 . Esto proporciona el alma más esbelta posible, que es tanto como decir el área mínima, puesto que se ha visto (Figura 2) que, para un área dada, $I$ y $W$ son mayores cuanto más esbelta sea el alma. Si no se comprueba el valor de $\beta$ se corre el riesgo de que el alma resultante sea demasiado esbelta, lo que invalida la hipótesis de estar en clase 3 (y, con ella, el predimensionamiento), o, por el contrario, de que sea demasiado gruesa, lo que lleva a un exceso de material.

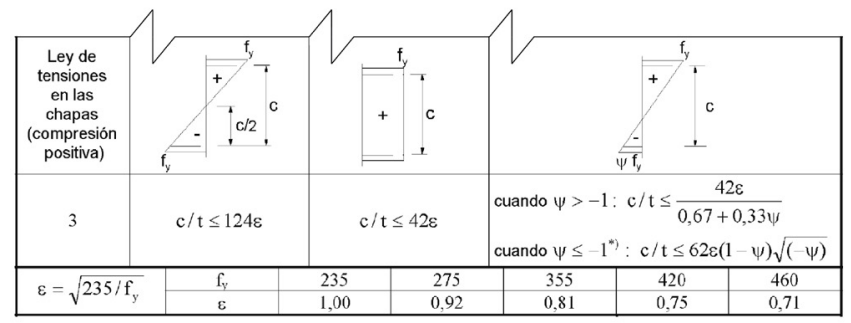

Figura 16. Criterios de clasificación para clase 3 según EC3 (12).

El proceso en la práctica es el siguiente: a) Con el valor inicial elegido de $\beta$ se obtiene el punto de área mínima de acuerdo simultáneamente con uno o varios de los criterios que decida el proyectista, como los mostrados en este artículo. b) Para ese punto solución se obtiene el valor de $t / c$ mínimo necesario para que la sección sea de clase 3. c) Tomando ahora como valor de $\beta$ el valor de $t / c$ mínimo recién obtenido se repite el cálculo del punto de área mínima. d) Se repiten los pasos b) y c) hasta que $\beta$ es igual o ligeramente superior al valor $t / c$ mínimo, es decir, hasta la convergencia. El proceso no es directo porque el valor de $t / c$ mínimo de una iteración depende del valor de $\beta$ de la iteración anterior, pero converge muy rápidamente.

Este criterio de clase de sección introduce una novedad: hasta ahora, todos los criterios se han representado en el mismo plano $(\Omega, z)$ para un valor $\beta=$ cte predefinido. Sin embargo, al iterar el valor de $\beta$ se trabaja en un plano $(\Omega, z)$ paralelo al anterior y diferente para cada iteración. El proceso de iteración se vuelve tridimensional, pero se conserva la aproximación intuitiva, pues toda la formulación depende sólo de las tres variables $\Omega, z$ y $\beta$.

A continuación, se muestra la aplicación del método para una sección de acero S275, para unos esfuerzos concomitantes de $N=-400 \mathrm{kN}$ (compresión) y $M=1000 \mathrm{kN} \cdot \mathrm{m}$. La inercia mínima ha de verificar $I_{\min }=0.002 \mathrm{~m}^{4}$, y el valor absoluto de la tensión máxima es $\sigma_{\max } \leq 240 \mathrm{MPa}$. En este caso no se aplica el criterio de limitación de cortante máximo. Para la primera iteración, mostrada en la Figura 17, se supone $\beta=1 / 100$. Aunque el algoritmo da una solución aparentemente correcta, la solución no es válida puesto que el punto óptimo, marcado por el cuadrado en la isolínea $\sigma_{\max }=240$, queda por encima de la isolínea $t / c \geq 0.01$ y casi por encima de la isolínea $t /$ $c \geq 0.0105$, muy cerca del valor $t / c \geq 0.0106$, que es la esbeltez mínima para que el alma pueda considerarse clase 3 .

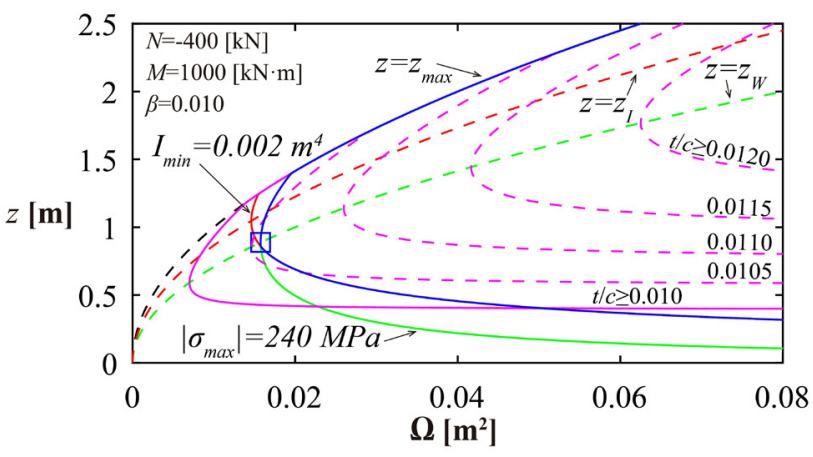

Figura 17. Ejemplo 2. Primera iteración $\beta=0.01$.

Repitiendo ahora los cálculos tomando $\beta=0.0106$ (Figura 18), se obtiene $\Omega=160.4 \mathrm{~cm}^{2}$ y $z=87.1 \mathrm{~cm}$, para una $t / c \geq 0.0105$, con lo que $\beta \geq t / c$ mínima para clase 3 .

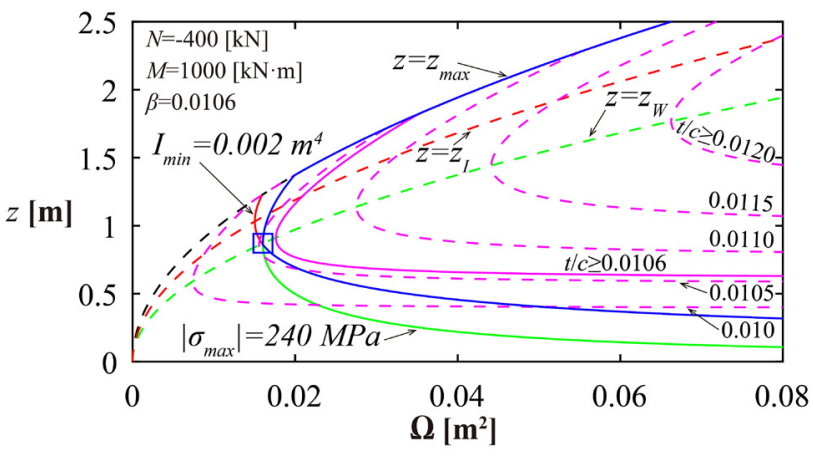

Figura 18. Ejemplo 2. Última iteración $\beta=0.0106$. 
Es de destacar que, tal y como se ha planteado, el método queda del lado de la seguridad al calcular la clase 3, pues se exige que $\beta$ (es $\operatorname{decir} \beta=t_{w} / z \approx t_{w} / h_{f}$ ), sea mayor o igual que $t / c$, sin considerar el hecho de que $z$ es sistemáticamente mayor que $c$, como se muestra en la Figura 15, puesto que no se consideran los espesores de las soldaduras ala-alma ni los dos semiespesores de las alas. Una vez definidos estos, el cálculo aún podría refinarse un poco más, si bien no hacerlo deja un leve margen de seguridad adicional al predimensionamiento.

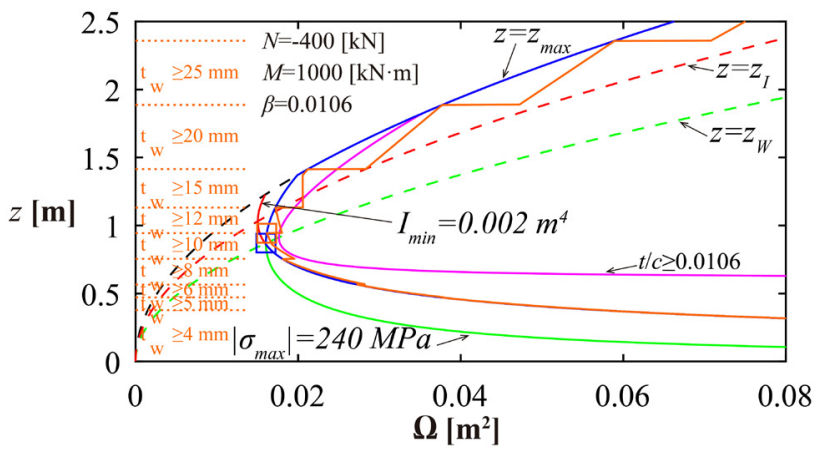

Figura 19. Ejemplo 2. Última iteración $\beta=0.0106$, incluyendo espesores de chapa comercial.

Sin embargo, el punto óptimo puede cambiar al tener que emplear chapas de espesor comercial por razones constructivas. El exceso de área por este motivo puede estimarse como $\left(t_{c}-\beta \cdot z\right) \cdot z$, donde, para un valor $z, t_{c}$ es el espesor comercial de la chapa inmediatamente superior al teórico del alma, $\beta \cdot z$. El sobrepeso será menor cuanto menos exceda el espesor comercial al teórico. Para ayudar al diseño final, la Figura 19 incorpora, para el valor de $\beta$, los límites de validez de cada espesor comercial de chapa. La curva azul de validez de todos los criterios se sustituye por la curva naranja, modificada por los espesores comerciales.

En la Figura 20 se muestra un detalle del predimensionamiento de la solución y los criterios empleados en este ejemplo. El punto óptimo teórico se ha obtenido combinando los criterios de inercia mínima y tensión longitudinal máxima. Se ha tomado un punto muy cercano a la isolínea de máxima esbeltez $t$ / $c \geq 0.106$ del alma para clase 3 . Si se considera el espesor comercial del alma, se obtiene la curva naranja, que coincide con la azul donde el espesor teórico coincide con uno comercial. El nuevo punto óptimo, es justo uno de esos puntos. Lógicamente, el área es mayor, de $161.4 \mathrm{~cm}^{2}$, si bien en este caso el incremento es muy pequeño.

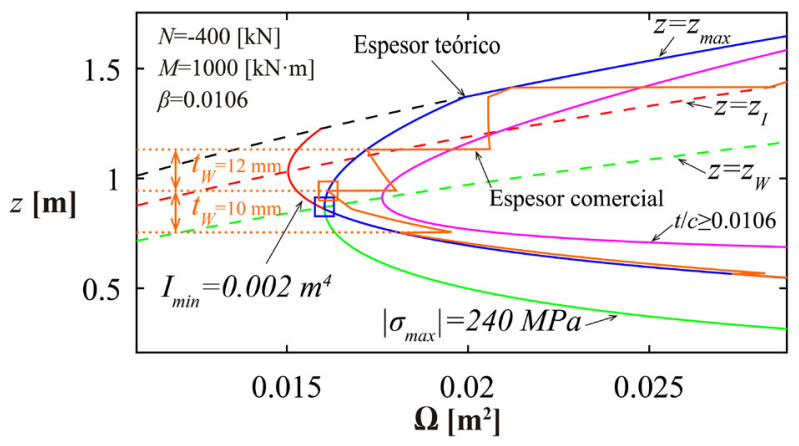

Figura 20. Detalle del entorno de la solución del ejemplo 2.

Las dimensiones de la sección óptima son pues $\Omega=161.4 \mathrm{~cm}^{2}$, $z=943 \mathrm{~mm}, t_{w}=\beta \cdot z=10.0 \mathrm{~mm}$. Las tensiones, medidas en los centros de las alas son $\sigma_{\text {sup }}=-239.97 \mathrm{MPa}$ y $\sigma_{\text {inf }}=+190.41 \mathrm{MPa}$, prácticamente igual al objetivo. En lo que se refiere a la clasificación de la sección, resulta, con $\psi=-0.7935$ y $\varepsilon=0.9244$, un valor de $c / t \leq 95.1$, que es cumplido por la sección, pues $c / t$ $\approx z / t_{w}=1 / \beta=94.3$, del lado de la seguridad pues este valor no considera el espesor de las alas ni las soldaduras, por lo que la sección es clase 3 .

\section{CONCLUSIONES}

En lo que se refiere al tratamiento simplificado de la sección, la notación empleada, en la que sólo hay tres variables significativas (el área, el canto y la esbeltez del alma), permite formular no sólo las características mecánicas de la sección, sino todas las expresiones necesarias para el predimensionamiento en el plano, con la suficiente precisión.

Este tratamiento simplificado permite, en diagramas gráficos que relacionan el área y el canto para valores constantes de la esbeltez del alma, determinar de un modo muy intuitivo, la sección transversal de peso mínimo, simultáneamente para una serie de criterios estructurales definidos por la normativa y la experiencia del proyectista.

En lo que respecta al predimensionamiento empleando simultáneamente los criterios de limitaciones de flechas y tensiones longitudinales máximas, se puede afirmar con carácter general, a la vista de los resultados, que para optimizar la sección es recomendable dar a la sección un canto entre $Z_{I}$ y $Z_{W}$ y más cercano al que condicione el criterio de dimensionamiento. El primero es el adecuado para un dimensionamiento en rigidez, pues maximiza la inercia, mientras que el segundo es el más adecuado para un dimensionamiento que limite la tensión longitudinal máxima, al maximizar el módulo elástico de la sección.

Sin embargo, durante la investigación realizada, se ha observado que los cantos de las secciones empleadas en proyectos reales suelen estar ligeramente por debajo de $z_{I}$ y $z_{W}$, bien sea por razones estéticas o funcionales. Cantos menores que éstos hacen que sea necesario aumentar el peso propio para proporcionar la misma resistencia a flexión y rigidez que con la sección de canto óptimo. Sin embargo, se ha observado, como se espera demostrar rigurosamente en futuras investigaciones, que el sobrepeso no es demasiado elevado (en torno a un 10\%) para secciones con canto ligeramente superiores al $70 \%$ de $z_{W}$ y al 80\% de $z_{I}$. Sólo si los cantos quedan muy por debajo de estos valores, se penaliza mucho el peso propio de la sección, en un fenómeno asimilable al que ocurre en vigas planas.

Es destacable el hecho de que la clasificación de la sección juega un papel fundamental en el resultado final de la optimización, así como la necesidad de utilizar chapas de espesor comercial.

Por último, se ruega al lector que disculpe a los autores por permitirse llamar la atención nuevamente sobre el hecho de que este método, como corresponde a cualquier estrategia de predimensionamiento, no cubre todos los posibles modos de fallo de la estructura, en particular aquellos que dependen del comportamiento en el plano perpendicular de la sección, como por ejemplo el pandeo lateral. Es obligación ineludible del proyectista conocer las limitaciones de los métodos que utiliza. 
Como líneas futuras de estudio pueden mencionarse el estudio de las secciones asimétricas, secciones mixtas, la definición de más criterios similares a los ya mostrados, la consideración de interacciones más complejas entre esfuerzos, o fenómenos como la inestabilidad local y la consideración de secciones eficaces.

Como último comentario sobre los métodos descritos en este artículo, se llama la atención sobre la importancia de la experiencia del proyectista, que resulta la única forma segura de obtener, durante el predimensionamiento, un equilibrio entre la complejidad del cálculo y la precisión de los resultados. En opinión de los autores, sólo un conocimiento profundo del tipo de estructura que se está proyectando permite definir adecuadamente, por ejemplo, aspectos como las acciones a considerar y sus factores de ponderación en las combinacio- nes simplificadas de cálculo, o las tensiones máximas que resultan razonables para ellas. Como se puede ver, este artículo ilustra, muy elocuentemente, la paradoja de que para realizar rápidamente cálculos sencillos suele ser necesario haber realizado con anterioridad numerosos cálculos rigurosos y afinados.

\section{AGRADECIMIENTOS}

Los autores agradecen a la Universidad Politécnica de Cartagena (UPCT, España) la financiación proporcionada a través del proyecto de investigación 2017_2420 dirigido por el primer autor. Además, los autores agradecen a la Fundación Séneca (Consejería de Industria, Turismo, Empresa e Innovación, Región de Murcia, España), la financiación de la beca de investigación (FPI) del segundo autor.

\section{REFERENCIAS}

(1) Dirección General de Carreteras (2000). Obras de paso de nueva construcción: Conceptos generales. Ministerio de Fomento. Centro de Publicaciones.

(2) Lebet, J.P; Hirt, M.A (2013). Steel Bridges. EPFL Press.

(3) Manterola Armisén, J. (2000). Puentes. Madrid.

(4) Menn, C. (1989). Prestressed Concrete Bridges. Birkhäuser Verlag.

(5) Leonhardt, F (1982). Bridges, Brücken. The Architectural Press.

(6) Salonga, J. y Gauvreau, P. (2014). Comparative Study of the Proportions, Form, and Efficiency of Concrete Arch Bridges. Journal of Bridge Engineering, 19(3), https://doi.org/10.1061/(ASCE)BE.1943-5592.0000537.

(7) Carbonell-Márquez, J.F., Gil-Martín L.M. y Hernández-Montes, E. (2013). Strength design optimization of structural steel members according to Eurocode 3. Journal of Constructional Steel Research, 8o, 213-223, https://doi.org/10.1016/j.jcsr.2012.07.019.

(8) Gil-Martín L.M., Aschheim M., y Hernández-Montes, E. (2008). Proportioning of Steel Beam-Column Members Based on RSD Methodology. Engineering Structures, 3O(11), 3003-3013, https://doi.org/10.1016/j.engstruct.2008.04.004.

(9) Dirección General de Carreteras (2012). Instrucción sobre las acciones a considerar en el proyecto de puentes de carretera. Ministerio de Fomento, Centro de publicaciones.

(10) Dirección General de Arquitectura, Vivienda y Suelo (2019). Código Técnico de la Edificación. Documento Básico SE Seguridad Estructural. Ministerio de Fomento. Madrid.

(11) UNE-EN 1991 (2019). Eurocódigo 1: Acciones en estructuras.

(12) UNE-EN 1993-1-1 (2013). Eurocódigo 3: Proyecto de estructuras de acero. Parte 1-1: Reglas generales y reglas para edificios.

(13) UNE-EN 1993-2 (2013). Eurocódigo 3: Proyecto de estructuras de acero. Parte 2: Puentes.

(14) Computers and Structures, Inc (2013). Analysis Reference Manual for SAP2Oooßv 16.

(15) Argüelles Álvarez, R (2005). Estructuras de acero: Cálculo, $2^{\mathrm{a}}$ edición. Bellisco, Ediciones Técnicas y Científicas. Madrid; 2005.

(16) García Guerrero, J.M. (2018). El puente arco espacial como una evolución tipológica. Universidad Politécnica de Cartagena. Tesis doctoral.

(17) Prendes Geroa, M.B., Bello García, A., y del Coz Díaz, J.J. (2006). Design optimization of 3D steel structures: Genetic algorithms vs. classical techniques. Journal of Constructional Steel Research, 62(12), 1303-1309, https://doi. org/10.1016/j.jcsr.2006.02.005.

(18) Liu, X., Yi, W.J., Li, Q.S., y Shen, P.S. (2008). Genetic evolutionary structural optimization. Journal of Constructional Steel Research, 64(3), 305-311, https://doi.org/10.1016/j.jcsr.2007.08.002.

(19) Saka, M.P., y Geem, Z.W. (2013). Mathematical and Metaheuristic Applications in Design Optimization of Steel Frame Structures: An Extensive Review. Mathematical Problems in Engineering, 2013, https://doi.org/10.1155/2013/271031

(20) Dirección General de Carreteras (1996). Recomendaciones para el proyecto de puentes metálicos para carreteras RPM-95. Ministerio de Fomento, Centro de publicaciones

(21) Dirección General de Carreteras (2003). Recomendaciones para el proyecto de puentes mixtos para carreteras RPX95. Ministerio de Fomento, Centro de publicaciones

(22) The MathWorks Inc (2017). Matlab (R2017b). 